\title{
Assessment of Economic Heterosis in Dual Purpose Sorghum [Sorghum bicolor (L.) Moench]
}

\author{
B.L. Meena ${ }^{1}$, B.R. Ranwah ${ }^{2}$, S.P. Das, H.S. Meena ${ }^{1}$, S.K. Meena, \\ R. Kumari and Anamika Nath ${ }^{2}$ \\ ${ }^{1}$ ICAR-DRMR, Bharatpur Rajasthan-321303, India \\ ${ }^{2}$ MPUAT, Rajasthan College of Agriculture, Udaipur Rajasthan -313001, India \\ *Corresponding author
}

\section{A B S T R A C T}

\begin{tabular}{|c|}
\hline Keywords \\
\hline $\begin{array}{l}\text { Economic heterosis, } \\
\text { Dual purpose } \\
\text { sorghum, } \\
\text { Multilocation } \\
\text { testing }\end{array}$ \\
\hline Article Info \\
\hline $\begin{array}{l}\text { Accepted: } \\
\text { 24 June } 2018 \\
\text { Available Online: } \\
\text { 10 July } 2018\end{array}$ \\
\hline
\end{tabular}

10 lines and 3 tester using L x T mating design consisted of 46 entries including 10 lines, 3 testers, 30 hybrids and three checks viz., CSV 23, CSV 27 and CSH25. These were evaluated in RBD with three replications during kharif 2015 in four environments viz., $22.5 \times 5 \mathrm{~cm}\left(\mathrm{E}_{1}\right), 30 \times 10 \mathrm{~cm}\left(\mathrm{E}_{2}\right), 45 \times 10 \mathrm{~cm}\left(\mathrm{E}_{3}\right)$ and $60 \times 10 \mathrm{~cm}\left(\mathrm{E}_{4}\right)$ at Rajasthan college of Agriculture, Udaipur in RBD with three replications. 24 crosses exhibited economic heterosis. Therefore these crosses may be identified as superior crosses for these characters. Three crosses $\mathrm{L}_{2} \times \mathrm{T}_{3}, \mathrm{~L}_{6} \times \mathrm{T}_{3}$ and $\mathrm{L}_{1} \times \mathrm{T}_{3}$ having economic heterosis for grain yield in $\mathrm{E}_{2}$. Where cross $\mathrm{L}_{2} \times \mathrm{T}_{3}$ and $\mathrm{L}_{6} \times \mathrm{T}_{3}$ also having economic heterosis for dry fodder yield in $E_{2}$. These crosses had good SCA effects and involving at least one good general combiner parent. This indicates superiority of $\mathrm{F}_{1}$ on account of accumulation of dominant genes. Two dual purpose crosses ICSA $29004 \times \mathrm{SPV} 1822\left(\mathrm{~L}_{2} \times \mathrm{T}_{3}\right)$ and ICSA $29012 \times$ SPV $1822\left(\mathrm{~L}_{6} \times \mathrm{T}_{3}\right)$ were identified for multilocation testing as these were having economic heterosis more than 15 per cent for grain yield and dry fodder yield. Grain purpose cross ICSA $29003 \times$ SPV $1822\left(\mathrm{~L}_{1} \times \mathrm{T}_{3}\right)$ is also identified for multilocation testing as it had very high economic heterosis for grain yield $(56.65 \%)$ in medium spacing environment i.e. $30 \times 10 \mathrm{~cm}$ along with good nicking in flowering and taller male parent.

\section{Introduction}

Sorghum bicolor (L.) Moench $(2 \mathrm{n}=20)$, family poaceae is one of the most important crops in the world because of its adaptation to a wide range of ecological conditions, suitability for low input cultivation and diverse uses (Doggett, 1988).

Sorghum green fodder is one of the cheapest sources of feed for milch, meat and draft animals. Among the cereals, sorghum plays an important role being grain cum fodder crop. Mainly three type of sorghum is cultivated i.e. grain, fodder and multicut sorghum. Grain sorghum is having low plant height and high harvest index, fodder sorghum having tall plants and multicut is leafy, thin stem and more tillering ability.

L $x$ T mating designs was used the estimation for economic heterosis in present investigation 
and information to be derived. Maintenance of plant population in per unit area is very difficult. Buffering ability of the genotypes is the only way to cope up with the available space. Therefore, breeding for buffering ability is another important aspect in genetic improvement of crop plants. Development of such a hybrid/variety, which gives a constant and desirable performance over wide range of spacing, is needed. For this, it is desirable to see the impact of various spacing on the yield of sorghum genotypes and identification of genotypes having buffering ability.

Hybrid vigor and its commercial exploitation have paid rich dividends in kharif sorghum leading to quantum jump in sorghum production. However, still it is far below in comparison to maize and pearl millet therefore there is a need for critical studies on combining ability and heterosis involving diverse source of male sterile lines and $\mathrm{R}$ lines.

In view of the above facts, present investigation entitled "Assessment of Economic Heterosis in Dual Purpose Sorghum [Sorghum bicolor (L.) Moench]', was planned and genotypes were evaluated during kharif, 2014 and kharif, 2015 at Instructional Farm, Rajasthan college of Agriculture,

\section{Materials and Methods}

The present investigation entitled "Assessment of Economic Heterosis in Dual Purpose Sorghum [Sorghum bicolor (L.) Moench]", was conducted at Instructional farm, Rajasthan College of Agriculture, Udaipur during kharif 2014 and kharif 2015. On the basis of days to flowering and suitability for dual purpose 36 lines were received from ICRISAT. After evaluation at this station 10 lines were identified on the basis of nicking of flowering. Three testers were identified on the basis of availability of restorer gene and past performance. Checks CSV 23, CSV 27 and CSH 25 were national checks in coordinated trials.

The experimental material comprised of 10 male sterile lines viz., ICSA $29003\left(\mathrm{~L}_{1}\right)$, ICSA $29004\left(\mathrm{~L}_{2}\right)$, ICSA $29006\left(\mathrm{~L}_{3}\right)$, ICSA 29010 $\left(\mathrm{L}_{4}\right)$, ICSA $29011\left(\mathrm{~L}_{5}\right)$, ICSA $29012\left(\mathrm{~L}_{6}\right)$, ICSA $29013\left(\mathrm{~L}_{7}\right)$, ICSA $29014\left(\mathrm{~L}_{8}\right)$, ICSA $29015\left(\mathrm{~L}_{9}\right)$ and ICSA 29016 (L 10), three restorer testers viz., SPV $245\left(\mathrm{~T}_{1}\right)$, SPV 1430 $\left(\mathrm{T}_{2}\right)$ and SPV $1822\left(\mathrm{~T}_{3}\right)$ and three checks viz. CSV 23, CSV 27, and CSH 25. These 10 lines and three testers were crossed in factorial fashion to obtain the 30 hybrids. The crossing programme was attempted at Udaipur during kharif 2014 and at Warangal during rabi 201415.In this experiment total 46 genotypes (10 lines, 3 testers, 30 crosses and 3 checks) were grown in a randomized block design with three replications in four different environments during kharif 2015 at Instructional farm, Rajasthan College of Agriculture, Udaipur (Rajasthan).

Each genotype was sown in a single row plot of 2 meter length maintaining a separate crop geometry (spacing) for each environments. The row to row and plant to plant spacing was $22.5 \mathrm{~cm} \times 5 \mathrm{~cm}, 30 \mathrm{~cm} \times 10 \mathrm{~cm}, 45 \mathrm{~cm} \times 10$ $\mathrm{cm}$ and $60 \mathrm{~cm} \times 10 \mathrm{~cm}$ in $\mathrm{E}_{1}, \mathrm{E}_{2}, \mathrm{E}_{3}$ and $\mathrm{E}_{4}$, respectively observations were recorded on days to $50 \%$ flowering, days to maturity, plant height $(\mathrm{cm})$, green fodder yield $\left(\mathrm{q} \mathrm{ha}^{-1}\right)$, dry fodder yield $\left(\mathrm{q} \mathrm{ha}{ }^{-1}\right)$, stay greenness $(0-1$ scale), ear head length $(\mathrm{cm})$, number of primaries per plant, number of seeds per primaries, seed index, harvest index $(\%)$,grain yield $\left(\mathrm{q} \mathrm{ha} \mathrm{h}^{-1}\right)$, protein content in fodder $(\%)$ and protein content in grain (\%). To record different observation five competitive plants in each plot were tagged at random. Plot means of all the characters were subjected to various statistical analysis except stay greenness. 


\section{Analysis of variance}

The plot means of each character were subjected to analysis of variance for individual environment as well as over the environment where error variance in different environment were homogeneous using least square technique of Fisher (1932).

\section{Economic heterosis}

Economic heterosis, expressed as per cent deviation toward desirable direction over standard check. Economic heterosis were calculated according to the method suggested Meredith and Bridge (1972) for individual as well as over the environments where mean square due to crosses were significant. Whereas, over the environment heterosis was calculated where error variance was homogeneous and mean square due to crosses were significant.

Economic heterosis $(\%)=\frac{\left(\overline{F_{1}}-\overline{B C}\right)}{\overline{B C}} \times 100$

It's significance was tested by using student ' $\mathrm{t}$ ' test.

$$
\begin{gathered}
t_{E D F}=\frac{\overline{F_{1}}-\overline{B C}}{S E_{\left(\overline{F_{1}}-\overline{B C}\right)}} \\
S E_{\left(\overline{F_{1}}-\overline{B C}\right)}=\sqrt{\frac{2 M S E}{n}}
\end{gathered}
$$

Where,

$$
\begin{aligned}
\overline{F_{1}}= & \text { Mean value of hybrid } \\
\overline{B C}= & \text { Mean value of best check } \\
\mathrm{n}= & \text { Divisor in respective conditions i.e. } \\
& \mathrm{r} \text { in case of individual environment } \\
& \text { and rs in case of over the } \\
& \text { environments. } \\
\mathrm{r}, \mathrm{s}= & \text { Number of replications and } \\
& \text { environments, respectively. } \\
\mathrm{MSE}= & \text { Error mean square for individual } \\
& \text { and over the environments, } \\
& \text { respectively. } \\
\mathrm{t}_{\mathrm{EDF}}= & \begin{array}{l}
\text { Student's ' } \mathrm{t} \text { ' at error degree of } \\
\text { freedom }
\end{array}
\end{aligned}
$$

To calculate economic heterosis parent and check had higher mean values were considered desirable for all the characters except traits like days to 50 per cent flowering and days to maturity where lower mean value was considered desirable and economic heterosis was calculated in desirable directions only.

\section{Results and Discussion}

The magnitude of economic heterosis expressed as per cent increase or decrease of $F_{1}$ value standard check (standard heterosis or economic heterosis) for various trait under different environments and pooled over the environment are presented in table 1 to 4 . The character wise results are summarized in following paragraphs:

\section{Days to 50 flowering}

Analysis of variances revealed significant difference between crosses in all the four environments. The mean square parents vs crosses were significant in $\mathrm{E}_{1}, \mathrm{E}_{2}, \mathrm{E}_{3}$ and $\mathrm{E}_{4}$. The economic heterosis was significant for $\mathrm{L}_{8}$ x $\mathrm{T}_{1}(-11.94 \%)$ and $\mathrm{L}_{4} \times \mathrm{T}_{2}(-17.41 \%)$ in $\mathrm{E}_{1}$; $\mathrm{L}_{8} \times \mathrm{T}_{1}(-8.28 \%)$ in $\mathrm{E}_{2}$ and $\mathrm{L}_{2} \times \mathrm{T}_{1}(-8.22 \%)$, $\mathrm{L}_{4} \times \mathrm{T}_{1}(-10.96 \%), \mathrm{L}_{5} \times \mathrm{T}_{1}(-5.94 \%), \mathrm{L}_{7} \times \mathrm{T}_{1}$ $(-10.05 \%), \mathrm{L}_{8} \times \mathrm{T}_{1}(-14.61 \%), \mathrm{L}_{9} \times \mathrm{T}_{1}(-5.48$ $\%), \mathrm{L}_{5} \times \mathrm{T}_{2}(-9.59 \%), \mathrm{L}_{6} \times \mathrm{T}_{2}(-14.16 \%), \mathrm{L}_{8} \times$ $\mathrm{T}_{2}(-14.16 \%), \mathrm{L}_{10} \times \mathrm{T}_{2}(-7.31 \%), \mathrm{L}_{4} \times \mathrm{T}_{3}(-$ $10.05 \%), \mathrm{L}_{6} \times \mathrm{T}_{3}(-5.94 \%), \mathrm{L}_{7} \times \mathrm{T}_{3}(-9.13 \%)$ and $\mathrm{L}_{8} \times \mathrm{T}_{3}(-13.24 \%)$ in $\mathrm{E}_{3}$ (Table 1).

\section{Days to maturity}

Analysis of variances revealed significant difference between crosses in all the four environments.

The $\mathrm{P}$ Vs $\mathrm{C}$ was significant in $\mathrm{E}_{1}, \mathrm{E}_{2}, \mathrm{E}_{3}$ and $\mathrm{E}_{4}$. Economic heterosis was not significant in any of the cross (Table 1). 


\section{Plant height}

Difference between crosses and P Vs C were significant in all the four environments and over the environments.

The economic heterosis was significant for 3 and 1 crosses in $E_{2}$ and $E_{3}$ respectively. Crosses exhibited significant economic heterosis were $\mathrm{L}_{3} \times \mathrm{T}_{3}(21.88 \%), \mathrm{L}_{5} \times \mathrm{T}_{3}$ $(18.01 \%)$ and $\mathrm{L}_{7} \times \mathrm{T}_{3}(12.19 \%)$ in $\mathrm{E}_{2}$ and $\mathrm{L}_{6}$ $x \mathrm{~T}_{3}(12.50 \%)$ in $\mathrm{E}_{3}$ (Table 1).

\section{Green fodder yield $\left(\mathbf{q} \mathrm{ha}^{-1}\right)$}

Analysis of variances revealed significant difference between crosses in all the four environments.

The $\mathrm{P}$ Vs $\mathrm{C}$ were significant in $\mathrm{E}_{2}, \mathrm{E}_{3}$ and $\mathrm{E}_{4}$.Economic heterosis was significant for $\mathrm{L}_{3} \mathrm{X}$ $\mathrm{T}_{3}(20.64 \%), \mathrm{L}_{5} \times \mathrm{T}_{3}(26.81 \%)$ and $\mathrm{L}_{10} \times \mathrm{T}_{3}$ $(17.75 \%)$ in $\left(\mathrm{E}_{2}\right)$ only.

Maximum economic heterosis was 26.81 per cent $\left(\mathrm{L}_{5} \times \mathrm{T}_{3}\right.$ in $\left.\mathrm{E}_{2}\right)$ (Table 2).

\section{Dry fodder yield (q ha $\left.{ }^{-1}\right)$}

Analysis of variances revealed significant difference between crosses in all the four environments. The $\mathrm{P}$ Vs $\mathrm{C}$ were significant in $\mathrm{E}_{1}, \mathrm{E}_{2}, \mathrm{E}_{3}$ and $\mathrm{E}_{4}$. The economic heterosis was significant in $\mathrm{E}_{2}$ only. Crosses exhibited significant economic heterosis in $\mathrm{E}_{2}$ were $\mathrm{L}_{3} \mathrm{x}$ $\mathrm{T}_{3}(16.63 \%)$ and $\mathrm{L} 6 \times \mathrm{T}_{3}(37.97 \%)$. (Table 2)

\section{Ear head length}

Analysis of variances revealed significant difference between crosses and $\mathrm{P}$ Vs $\mathrm{C}$ for all the four environments and over the environments.

Economic heterosis was not significant in any cross in any environment. (Table 2)

\section{Number of primaries per plant}

Analysis of variances revealed significant difference between crosses in all the four environments. The $\mathrm{P}$ Vs $\mathrm{C}$ were significant in $\mathrm{E}_{2}, \mathrm{E}_{3}$ and $\mathrm{E}_{4}$. Economic heterosis was not significant in any cross in any environment (Table 3).

\section{Number of seeds per primary}

Analysis of variances revealed significant difference between crosses and $\mathrm{P}$ Vs $\mathrm{C}$ in all the four environments. Economic heterosis was significant for $\mathrm{L}_{8} \times \mathrm{T}_{1}(11.72 \%), \mathrm{L}_{4} \times \mathrm{T}_{2}$ (27.43\%) and $\mathrm{L}_{7} \times \mathrm{T}_{3}(11.47 \%)$ in $\mathrm{E}_{1}, \mathrm{~L}_{7} \times \mathrm{T}_{2}$ (39.18\%) in $\mathrm{E}_{2}$ and $\mathrm{L}_{2} \times \mathrm{T}_{1}(19.68 \%), \mathrm{L}_{4} \times \mathrm{T}_{2}$ (34.54\%), $\mathrm{L}_{2} \times \mathrm{T}_{3}(15.66 \%), \mathrm{L}_{4} \times \mathrm{T}_{3}(36.95$ $\%)$ in $\mathrm{E}_{3}$ (Table 3)

\section{Seed index}

Analysis of variances revealed significant difference between crosses and $\mathrm{P}$ Vs $\mathrm{C}$ in all the four environments. Economic heterosis was significant for 3 crosses viz., $\mathrm{L}_{3} \times \mathrm{T}_{1}$ (7.69), $\mathrm{L}_{3} \times \mathrm{T}_{2}$ (5.86) and $\mathrm{L}_{5} \times \mathrm{T}_{3}$ (4.30) only in $\mathrm{E}_{4}$ (Table 3)

\section{Harvest index}

Analysis of variances revealed significant difference between crosses in $\mathrm{E}_{3}$ and $\mathrm{E}_{4}$ only and $\mathrm{P}$ Vs $\mathrm{C}$ was non-significant in all the four environments.

The heterosis for harvest index ranged from $46.10\left(\mathrm{~L}_{10} \times \mathrm{T}_{1}\right)$ to -53.75 per cent $\left(\mathrm{L}_{3} \times \mathrm{T}_{3}\right)$ in $\mathrm{E}_{3}$ and $26.54\left(\mathrm{~L}_{7} \times \mathrm{T}_{3}\right)$ to -14.24 per cent $\left(\mathrm{L}_{1} \mathrm{X}\right.$ $\mathrm{T}_{2}$ ) in $\mathrm{E}_{4}$. Positive heterosis was significant for 8 crosses in $E_{3}$ and 6 crosses in $E_{4}$ where as negative heterosis was significant for 3 crosses in $\mathrm{E}_{3}$ and 5 crosses in $\mathrm{E}_{4}$. Heterobeltiosis was significant for cross $\mathrm{L}_{10} \times \mathrm{T}_{1}(36.80 \%)$ in $\mathrm{E}_{3}$ and $\mathrm{L}_{2} \times \mathrm{T}_{1}(10.33 \%)$ crosses in $\mathrm{E}_{4}$. Economic heterosis was not significant in any cross in any environment (Table 4). 


\section{Grain yield}

Difference between crosses and P Vs C were significant in all the four environments. Economic heterosis was significant for 3 crosses viz., $\mathrm{L}_{1} \times \mathrm{T}_{3}(56.65 \%), \mathrm{L}_{2} \times \mathrm{T}_{3}(15.27$ $\%)$ and $\mathrm{L}_{6} \times \mathrm{T}_{3}(20.20 \%)$ in $\mathrm{E}_{2}$ only (Table 4)

\section{Protein content in grain}

Difference between crosses and P Vs C were significant in all the four environments.
Economic heterosis was significant for $\mathrm{L}_{1} \times \mathrm{T}_{2}$ $(9.49 \%)$ cross in $\mathrm{E}_{3}$ only (Table 4)

\section{Protein content in fodder}

Difference between crosses and P Vs C were significant in all the four environments. Economic heterosis was significant for 2 crosses viz., $\mathrm{L}_{2} \times \mathrm{T}_{3}(8.34 \%)$ and $\mathrm{L}_{4} \times \mathrm{T}_{3}(6.66$ $\%)$ in $\mathrm{E}_{1}$ (Table 4)

Table.1 Economic heterosis for days to 50\% flowering, days to maturity and plant height

\begin{tabular}{|c|c|c|c|c|c|c|c|c|c|c|c|c|c|}
\hline \multirow{2}{*}{$\begin{array}{l}\text { S. } \\
\text { No. }\end{array}$} & \multirow[t]{2}{*}{ Crosses } & \multicolumn{4}{|c|}{ Days to $50 \%$ flowering } & \multicolumn{4}{|c|}{ Days to maturity } & \multicolumn{4}{|c|}{ Plant height } \\
\hline & & E1 & E2 & E3 & E4 & E1 & E2 & $\mathbf{E 3}$ & E4 & E1 & E2 & $\mathbf{E 3}$ & E4 \\
\hline 1. & L1 $\times$ T1 & - & - & -0.91 & - & - & - & - & - & - & - & - & - \\
\hline 2. & L2 $\times$ T1 & - & - & $-8.22 * *$ & - & 0.00 & - & - & - & - & 2.49 & - & - \\
\hline 3. & L3 $\times$ T1 & - & - & - & - & - & - & - & - & - & - & - & - \\
\hline 4. & L4 $\times$ T1 & - & - & $-10.96^{* *}$ & - & - & - & - & - & - & - & - & - \\
\hline 5. & L5 $\times$ T1 & - & -0.52 & $-5.94 *$ & - & $-3.61 * *$ & - & - & - & - & - & - & - \\
\hline 6. & L6 $\times$ T1 & - & - & -4.57 & - & $-3.53 * *$ & - & - & - & - & - & - & - \\
\hline 7. & L7 $\times$ T1 & - & - & $-10.05 * *$ & - & $-4.75 * *$ & - & - & - & - & - & - & - \\
\hline 8. & L8 $\times$ T1 & $-11.94 *$ & - & $-14.61 * *$ & -1.08 & $-7.28 * *$ & - & - & - & - & - & - & - \\
\hline 9. & L9 $\times$ T1 & - & - & $-5.48^{*}$ & - & -1.27 & - & - & - & - & - & - & - \\
\hline 10. & L10 x T1 & - & - & - & - & -0.32 & - & - & - & 2.00 & - & - & - \\
\hline 11. & L1 $\times$ T2 & - & - & - & - & - & - & - & - & - & - & - & - \\
\hline 12. & L2 $\times$ T2 & - & - & -2.74 & - & - & - & - & - & - & - & - & - \\
\hline 13. & L3 $\times$ T2 & - & - & 0.00 & - & - & - & - & - & - & - & - & - \\
\hline 14. & L4 $\times$ T2 & $-17.41 * *$ & - & -3.20 & - & - & - & - & - & - & - & - & - \\
\hline 15. & L5 $\times$ T2 & - & - & $-9.59 * *$ & - & - & - & - & - & - & - & - & - \\
\hline 16. & L6 $\times$ T2 & -0.50 & -1.55 & $-14.16^{* * *}$ & - & - & - & - & - & - & - & - & - \\
\hline 17. & L7 $\times$ T2 & -2.49 & - & -1.83 & - & -1.00 & - & - & - & - & - & - & - \\
\hline 18. & L8 $\times$ T2 & -9.45 & $-8.25 *$ & $-14.16^{* * *}$ & - & - & - & - & - & - & - & - & - \\
\hline 19. & L9 $\times$ T2 & - & - & - & - & - & - & - & - & - & - & - & - \\
\hline 20. & $\mathrm{~L} 10 \times \mathrm{T} 2$ & - & - & $-7.31 * *$ & - & $-6.33^{* *}$ & -1.07 & -1.06 & -0.35 & - & - & - & - \\
\hline 21. & L1 $\times$ T3 & - & - & - & - & $-4.25 * *$ & - & - & - & - & - & 7.03 & 2.60 \\
\hline 22. & L2 $\times$ T3 & - & - & - & - & -0.68 & - & - & - & - & 5.40 & - & - \\
\hline 23. & L3 $\times$ T3 & - & - & - & - & - & - & - & - & 1.33 & $21.88 * *$ & - & 2.05 \\
\hline 24. & L4 $\times$ T3 & - & - & $-10.05^{* * *}$ & - & $-4.58 * *$ & - & - & - & 1.47 & - & - & 2.46 \\
\hline 25. & L5 $\times$ T3 & - & - & - & - & $-3.28 * *$ & - & - & - & - & $18.01 * *$ & - & - \\
\hline 26. & L6 $\times$ T3 & - & - & $-5.94 *$ & - & -1.96 & - & - & - & - & 5.96 & $12.50^{*}$ & - \\
\hline 27. & L7 $\times$ T3 & - & - & $-9.13^{* *}$ & - & $-3.27 * *$ & - & - & - & 0.00 & $12.19^{*}$ & - & 0.00 \\
\hline 28. & L8 $\times$ T3 & - & 0.00 & $-13.24 * *$ & -5.38 & - & - & - & - & - & - & 1.56 & 3.55 \\
\hline 29. & L9 $\times$ T3 & - & - & - & - & - & - & - & - & - & - & - & - \\
\hline 30. & $\mathrm{~L} 10 \times \mathrm{T} 3$ & - & - & - & - & - & - & - & - & - & - & 4.69 & 10.79 \\
\hline
\end{tabular}

** Significant at 5 and 1 per cent, respectively. 
Table.2 Heterosis for green fodder yield, dry fodder yield and ear head length

\begin{tabular}{|c|c|c|c|c|c|c|c|c|c|c|c|c|c|}
\hline \multirow{2}{*}{$\begin{array}{c}\text { S. } \\
\text { No. }\end{array}$} & \multirow[t]{2}{*}{ Crosses } & \multicolumn{4}{|c|}{ Green fodder yield } & \multicolumn{4}{|c|}{ Dry fodder yield } & \multicolumn{4}{|c|}{ Ear head length } \\
\hline & & E1 & $\mathbf{E 2}$ & $\mathbf{E 3}$ & $\mathbf{E 4}$ & E1 & $\mathbf{E 2}$ & $\mathbf{E 3}$ & $\mathbf{E 4}$ & E1 & E2 & $\mathbf{E 3}$ & $\mathbf{E 4}$ \\
\hline 1. & L1 $\times$ T1 & - & - & - & - & - & - & - & - & - & - & - & - \\
\hline 2. & L2 $\times$ T 1 & - & - & - & - & - & - & - & - & - & - & - & - \\
\hline 3. & L3 $\times$ T 1 & - & - & - & - & - & - & - & - & - & - & - & - \\
\hline 4. & L4 $\times$ T1 & - & - & - & - & - & - & - & - & - & - & - & - \\
\hline 5. & L5 $\times$ T1 & - & - & - & - & - & - & - & - & - & - & - & - \\
\hline 6. & L6 $\times$ T1 & - & - & - & - & - & - & - & - & - & - & - & - \\
\hline 7. & L7 $\times$ T1 & - & - & - & - & - & - & - & - & - & - & - & - \\
\hline 8. & L8 $\times$ T1 & - & - & - & - & - & - & - & - & - & - & - & - \\
\hline 9. & L9 $\times$ T1 & - & - & - & - & - & - & - & - & - & - & - & - \\
\hline 10. & $\mathrm{~L} 10 \mathrm{x}$ T1 & - & - & - & - & - & - & - & - & - & - & - & - \\
\hline 11. & L1 $\times$ T2 & - & - & - & - & - & - & - & - & - & - & - & - \\
\hline 12. & L2 $\times$ T2 & - & - & - & - & - & - & - & - & - & - & - & - \\
\hline 13. & L3 $\times$ T2 & - & - & - & - & - & - & - & - & - & - & - & - \\
\hline 14. & L4 $\times$ T2 & - & - & - & - & - & - & - & - & - & - & - & - \\
\hline 15. & L5 $\times$ T2 & - & - & - & - & - & - & - & - & - & - & - & - \\
\hline 16. & L6 $\times$ T2 & - & - & - & - & - & - & - & - & - & - & - & - \\
\hline 17. & L7 $\times$ T2 & - & - & - & - & - & - & - & - & - & - & - & - \\
\hline 18. & L8 $\times$ T2 & - & - & - & - & - & - & - & - & - & - & - & - \\
\hline 19. & L9 $\times$ T2 & - & - & - & - & - & - & - & - & - & - & - & - \\
\hline 20. & $\mathrm{~L} 10 \times \mathrm{T} 2$ & - & - & - & - & - & 1.18 & - & - & - & - & - & - \\
\hline 21. & L1 $x$ T3 & - & - & - & - & - & - & 8.63 & - & - & - & - & - \\
\hline 22. & L2 $x$ T3 & - & - & - & 2.33 & - & $16.63 * *$ & - & 2.93 & - & - & - & 2.33 \\
\hline 23. & L3 $\times$ T3 & - & $20.64 * *$ & - & - & - & 10.26 & - & - & - & $20.64 * *$ & - & - \\
\hline 24. & L4 $\times$ T3 & - & - & - & - & - & - & - & - & - & - & - & - \\
\hline 25. & L5 $\times$ T3 & - & $26.81^{* *}$ & - & - & - & 0.71 & - & - & - & $26.81 * *$ & - & - \\
\hline 26. & L6 $x$ T3 & - & - & - & - & - & $37.97 * *$ & - & - & - & - & - & - \\
\hline 27. & L7 $x$ T3 & - & - & - & - & - & - & - & - & - & - & - & - \\
\hline 28. & L8 $\times$ T3 & - & - & - & - & - & - & - & - & - & - & - & - \\
\hline 29. & L9 $x$ T3 & - & - & - & - & - & - & - & - & - & - & - & - \\
\hline 30. & L10 x T3 & - & $14.75^{* *}$ & - & - & - & - & - & - & - & $14.75^{* *}$ & - & - \\
\hline
\end{tabular}

** Significant at 5 and 1 per cent, respectively. 
Table.3 Economic heterosis for number of primaries per plant, number of seeds per primary and Seed index

\begin{tabular}{|c|c|c|c|c|c|c|c|c|c|c|c|c|c|}
\hline \multirow[t]{2}{*}{ S. No. } & \multirow[t]{2}{*}{ Crosses } & \multicolumn{4}{|c|}{ Number of primaries per plant } & \multicolumn{4}{|c|}{ Number of seeds per primary } & \multicolumn{4}{|c|}{ Seed index } \\
\hline & & E1 & $\mathbf{E 2}$ & $\mathbf{E 3}$ & $\mathbf{E 4}$ & E1 & $\mathbf{E 2}$ & $\mathbf{E 3}$ & $\mathbf{E 4}$ & E1 & E2 & $\mathbf{E 3}$ & $\mathbf{E 4}$ \\
\hline 1. & $\mathrm{~L} 1 \times \mathrm{T} 1$ & - & - & - & - & - & - & - & - & - & - & - & - \\
\hline 2. & $\mathrm{~L} 2 \times \mathrm{T} 1$ & - & - & - & - & - & - & $19.68^{*}$ & - & - & - & - & - \\
\hline 3. & L3 $\times$ T1 & - & - & - & - & - & - & - & - & - & - & 8.35 & $7.69 * *$ \\
\hline 4. & $\mathrm{~L} 4 \times \mathrm{T} 1$ & - & - & - & - & - & - & - & - & - & - & - & - \\
\hline 5. & L5 $x$ T1 & - & - & - & - & - & - & - & - & - & - & - & 2.38 \\
\hline 6. & L6 $\times$ T1 & - & - & - & - & - & - & - & - & - & - & - & - \\
\hline 7. & L7 x T1 & - & - & - & - & - & 0.00 & - & - & - & - & - & - \\
\hline 8. & L8 $\times \mathrm{T} 1$ & - & - & - & - & $11.72 *$ & 3.27 & - & - & - & - & - & - \\
\hline 9. & L9 $\times$ T1 & - & - & - & - & 2.00 & - & - & - & - & - & - & - \\
\hline 10. & L10 x T1 & - & - & - & - & - & - & - & - & - & - & - & - \\
\hline 11. & $\mathrm{~L} 1 \times \mathrm{T} 2$ & - & - & - & - & - & - & - & - & - & - & - & - \\
\hline 12. & $\mathrm{~L} 2 \times \mathrm{T} 2$ & - & - & - & - & - & - & 0.80 & - & - & - & - & - \\
\hline 13. & L3 $\times$ T2 & - & - & - & - & - & - & - & - & - & - & - & $5.86^{* *}$ \\
\hline 14. & $\mathrm{~L} 4 \times \mathrm{T} 2$ & - & - & - & - & $27.43 * *$ & - & $34.54 * *$ & - & - & - & - & - \\
\hline 15. & L5 $\times$ T2 & - & - & - & 0.38 & - & - & 4.82 & - & - & - & - & - \\
\hline 16. & L6 $\times$ T2 & - & - & - & - & - & - & 8.03 & - & - & - & - & - \\
\hline 17. & L7 $\times$ T2 & - & - & - & - & - & $39.18 * *$ & 11.24 & - & - & - & - & - \\
\hline 18. & L8 $\times \mathrm{T} 2$ & - & - & - & - & - & - & - & - & - & - & - & - \\
\hline 19. & L9 $\times$ T2 & - & - & - & - & - & - & - & - & - & - & - & - \\
\hline 20. & $\mathrm{~L} 10 \times \mathrm{T} 2$ & - & - & - & - & - & - & - & - & - & - & - & - \\
\hline 21. & $\mathrm{~L} 1 \times \mathrm{T} 3$ & - & - & - & - & - & - & - & 0.77 & - & - & - & - \\
\hline 22. & $\mathrm{~L} 2 \times \mathrm{T} 3$ & - & - & - & - & - & 3.67 & $15.66^{*}$ & - & - & - & - & 0.82 \\
\hline 23. & L3 $\times$ T3 & - & - & - & - & - & - & - & - & - & - & 0.99 & 2.56 \\
\hline 24. & $\mathrm{~L} 4 \times \mathrm{T} 3$ & - & - & - & - & 6.48 & - & $36.95 * *$ & - & - & - & 0.83 & - \\
\hline 25. & L5 $\times$ T3 & - & - & 1.80 & - & - & - & - & - & - & - & - & $4.30 * *$ \\
\hline 26. & L6 $x$ T3 & - & - & - & - & - & - & - & - & - & - & - & - \\
\hline 27. & L7 $\times$ T3 & - & - & - & - & $11.47^{*}$ & - & - & - & - & - & - & - \\
\hline 28. & L8 $\times$ T3 & - & - & - & - & - & 7.76 & - & - & - & - & - & 0.82 \\
\hline 29. & L9 $x$ T3 & - & - & - & - & - & - & - & - & - & - & - & - \\
\hline 30. & $\mathrm{~L} 10 \times \mathrm{T} 3$ & - & - & - & - & - & - & - & - & - & - & - & - \\
\hline
\end{tabular}


Table.4 Economic heterosis for harvest index, grain yield, protein content in grain $(\mathrm{g})$ and protein content in fodder (f)

\begin{tabular}{|c|c|c|c|c|c|c|c|c|c|c|c|c|c|}
\hline \multirow{2}{*}{$\begin{array}{c}\text { S. } \\
\text { No. }\end{array}$} & \multirow[t]{2}{*}{ Crosses } & \multicolumn{2}{|c|}{ Harvest index } & \multicolumn{4}{|c|}{ Grain yield } & \multicolumn{4}{|c|}{ Protein content (g) } & \multicolumn{2}{|c|}{ Protein content (f) } \\
\hline & & E3 & E4 & E1 & E2 & E3 & E4 & E1 & E2 & E3 & E4 & E1 & E2 \\
\hline 1. & L1 $\times$ T1 & - & - & - & - & - & - & - & - & - & - & - & - \\
\hline 2. & L2 $x$ T1 & - & 0.44 & - & - & - & - & - & - & - & - & - & - \\
\hline 3. & L3 $x$ T1 & - & - & 2.62 & - & - & - & - & - & - & - & - & - \\
\hline 4. & L4 $\times$ T1 & - & - & - & - & - & - & - & - & - & - & - & - \\
\hline 5. & L5 $\times$ T1 & - & - & - & - & - & - & - & - & - & - & - & - \\
\hline 6. & L6 $\times$ T1 & - & - & - & - & 1.50 & - & - & - & - & - & - & - \\
\hline 7. & L7 $\times$ T1 & - & - & - & - & - & - & - & - & - & - & - & - \\
\hline 8. & L8 $\times$ T1 & - & - & - & - & - & - & - & - & - & - & - & - \\
\hline 9. & L9 $\times$ T1 & - & - & - & - & - & - & - & - & - & - & - & - \\
\hline 10. & $\mathrm{~L} 10 \times \mathrm{T} 1$ & 2.20 & - & - & - & - & - & - & - & - & - & 1.76 & - \\
\hline 11. & L1 $\times$ T2 & - & - & - & - & - & - & - & 2.12 & $9.49 * *$ & - & - & - \\
\hline 12. & L2 $\times$ T2 & - & - & - & - & - & - & - & - & - & - & - & - \\
\hline 13. & L3 $\times$ T2 & - & - & - & - & - & - & - & - & - & - & - & - \\
\hline 14. & $\mathrm{~L} 4 \times \mathrm{T} 2$ & - & - & - & - & - & - & - & - & - & - & - & - \\
\hline 15. & L5 $\times$ T2 & - & - & - & - & - & - & - & - & - & - & - & - \\
\hline 16. & L6 $\times$ T2 & - & - & - & - & - & - & - & - & - & - & - & - \\
\hline 17. & L7 $\times$ T2 & - & - & - & - & - & - & - & - & - & - & - & - \\
\hline 18. & L8 $\times$ T2 & - & - & - & - & - & - & - & - & - & - & - & - \\
\hline 19. & L9 $\times$ T2 & - & - & - & - & - & - & - & - & - & - & - & - \\
\hline 20. & $\mathrm{~L} 10 \times \mathrm{T} 2$ & - & - & - & - & - & - & - & - & - & - & - & - \\
\hline 21. & L1 1 T3 & - & - & - & $56.65^{* *}$ & - & - & - & - & - & - & - & - \\
\hline 22. & L2 $\times$ T3 & - & - & - & $15.27 * *$ & - & - & 0.94 & - & - & - & $8.34 * *$ & - \\
\hline 23. & L3 $\times$ T3 & - & - & - & 7.88 & - & - & - & - & - & - & - & - \\
\hline 24. & L4 $\times$ T3 & - & - & - & - & - & - & - & - & - & - & $6.66^{* * *}$ & - \\
\hline 25. & L5 $\times$ T3 & - & - & - & 2.96 & - & - & - & - & - & - & - & - \\
\hline 26. & L6 $\times$ T3 & - & - & - & $20.20 * *$ & - & - & - & - & - & - & - & - \\
\hline 27. & L7 $\times$ T3 & - & - & - & - & - & - & - & - & - & - & - & - \\
\hline 28. & L8 $\times$ T3 & - & - & - & - & - & - & - & - & - & - & - & - \\
\hline 29. & L9 $\times$ T3 & - & - & - & - & - & - & - & - & - & - & - & - \\
\hline 30. & L10 $\times$ T3 & - & - & - & - & - & - & - & - & - & - & - & - \\
\hline
\end{tabular}

** Significant at 5 and 1 per cent, respectively. 
Economic heterosis was significant for nine characters in one or more environments but, different crosses exhibited economic heterosis for different characters. Maximum number of economic heterotic crosses were observed for days to flowering (17) followed by number of seeds per primary (8), plant height (6), grain yield (3), green fodder yield (3), seed index (3), dry fodder yield (2), protein content in fodder (2) and protein content in grain (1). The crosses exhibited economic heterosis in more than one environment were $\mathrm{L}_{8} \times \mathrm{T}_{1}$, in $E_{1}$ and $E_{3}$ and $L_{8} \times T_{2}$ in $E_{2}$ and $E_{3}$ for days to flowering; $L_{4} \times T_{2}$ in $E_{1}$ and $E_{3}$ for number of seeds per primary; $L_{3} \times T_{3}$ in $E_{2}$ and pool for plant height and $\mathrm{L}_{2} \times \mathrm{T}_{3}$ in $\mathrm{E}_{1}, \mathrm{E}_{2}$ and $\mathrm{E}_{3}, \mathrm{~L}_{4} \mathrm{x}$ $\mathrm{T}_{3}$ in $\mathrm{E}_{1}$ and $\mathrm{E}_{3}, \mathrm{~L}_{5} \times \mathrm{T}_{3}$ in $\mathrm{E}_{2}$ and $\mathrm{E}_{4}, \mathrm{~L}_{6} \times \mathrm{T}_{3}$ in $E_{2}$ and $E_{3}$ and $L_{7} \times T_{3}$ in $E_{1}, E_{2}, E_{3}$ and pool for different characters. There were eight crosses exhibited economic heterosis for two characters in an environment viz., $\mathrm{L}_{2} \times \mathrm{T}_{1}$ $\left(\mathrm{E}_{3}\right), \mathrm{L}_{8} \times \mathrm{T}_{1}\left(\mathrm{E}_{1}\right), \mathrm{L}_{4} \times \mathrm{T}_{2}\left(\mathrm{E}_{1}\right)$ and $\mathrm{L}_{4} \times \mathrm{T}_{3}$ $\left(E_{3}\right)$ for days to flowering and number of seed per primary, $L_{3} \times T_{3}\left(E_{2}\right)$ and $L_{5} \times T_{3}\left(E_{2}\right)$ for plant height and green fodder yield and $\mathrm{L}_{2} \mathrm{X}$ $\mathrm{T}_{3}\left(\mathrm{E}_{2}\right), \mathrm{L}_{6} \times \mathrm{T}_{3}\left(\mathrm{E}_{2}\right)$ for grain yield and dry fodder yield. Cross $\mathrm{L}_{6} \times \mathrm{T}_{3}$ also exhibited economic heterosis for plant height and early flowering in $\mathrm{E}_{3}$. This indicates that for grain yield three crosses viz., $\mathrm{L}_{1} \times \mathrm{T}_{3}, \mathrm{~L}_{2} \times \mathrm{T}_{3}$ and $\mathrm{L}_{6}$ $\mathrm{x} \mathrm{T}_{3}$ exhibited economic heterosis out of them two crosses viz., $\mathrm{L}_{2} \times \mathrm{T}_{3}$ and $\mathrm{L}_{6} \times \mathrm{T}_{3}$ were also heterobeltiosis for dry fodder yield in $E_{2}$ that is at spacing $30 \times 10 \mathrm{~cm}$.

Apart from above crosses cross $\mathrm{L}_{6} \times \mathrm{T}_{3}$ had economic heterosis for days to $50 \%$ flowering and plant height in $\mathrm{E}_{3}$ and for dry fodder yield in $E_{2}$ and $L_{2} \times T_{3}$ for dry fodder yield in $E_{2}$, protein content in fodder in $E_{1}$ and number of primaries per plant in $\mathrm{E}_{3}$ may be utilized for these characters.

\section{References}

Anonymous 2015. Directorate of Agriculture, Govt. of Rajasthan, Jaipur, Rajasthan.
Anonymous 2015. Agriculture Statistics At a Glance. Agriculture Statistics Division, Directorate of Economics and Statistics, Department of Agriculture and cooperation Ministry of Agriculture, Govt. of India, New Delhi.

Anonymous 2015. FAOSTAT, Food and Agriculture Organization of the United Nations Statistics Division, Rome.

Doggett H, 1988. Sorghum. Longman Scientific \& Technical, London Cereal Sci 44: 236-251

Fisher, R. A. (1932). The Genetical Theory of Natural Selection. Clarendon, Oxford.

Fisher, R.A. and Yates, F. (1967). Statistical tables for biological, agricultural and medical research. Longman Group Limited, London.

Fonesca, S. and Patterson, F. (1968). Hybrid vigour in seven parent diallel in winter wheat (Triticum aestivum L.). Crop Sci., 8:85-95.

Ganesh, S., Khan, A.K.F and Senthil, N. (1996). Heterosis studies for grain yield characters in sweet sorghum. Madras Agricultural Journal.83:655657.

Hariprasanna, K., Rajendrakumar, P. and Patil, J. V. (2012). Parental selection for high heterosis in sorghum [Sorghum bicolor (L.) Moench]combining ability, heterosis and their inter-relationships. Crop Research, 44:3, 400-408. Heredity, 9: 223-228.

Kulakarni, V., Salimath, P.M and Patil, M.S. (2007). Combining ability analysis in rabi sorghum [Sorghum bicolor (L.) Moench]. Crop Research., 32 (3): 455-458.

Meredith, W.R. and Bridge, R.R. (1972). Heterosis and gene action in cotton (Gossypium hirsutam). Crop Sci., 12: 304-310.

Murty, U. R. (1985). The concept of vybrid 
III. Stability of grain, biological and fodder yield of purelines, vybrids and hybrids in Sorghum bicolor (L.) Moench. Cereal Res. Communication, 13: 169-176.

Panse, V. G. and Sukhatme, P. V. (1985). Statistical Methods for Agricultural Workers. Indian Council of Agricultural Research, New Delhi.

Panse, V.G and Sukhatme, P.V. (1961). Statistical Methods. Oxford and IBH Publishers,

Salini, K., Satyanarayana Rao, V., Ramana, J.V and Srinivasa Rao, V. (2008). Line $\mathrm{x}$ tester analysis in dual purpose sorghum [Sorghum bicolor (L.) Moench]. The Andhra Agricultural Journal. 55 (2): 166-174.

Salunke, C.B. and Deore, G.N. (1998). Heterosis in rabi sorghum for grain yield and its components. Madras Agricultural Journal, 87 (1): 143-144.

Sharma, H., and Sharma, G. S. and Dadheech,
A. (2002). Heterosis for grain yield and its component traits in sorghum (Sorghum bicolor (L.) Moench). Ann. Agric. Res. New series, 24 (3): 579582.

Shull, G. H. (1914). Hybridization methods in corn breeding. American Breeder's Mav., 1: 98-107. In: Heterosis (Gowen, J. W. Ed.). Hafner Inc., New York, pp. 50.

Shull, G.H. (1914). Duplicate gene for capsule form in Capsella bursapustoris. Zeit Induk Abstr. V. Verbung. 12: 94-149. In "Heterosis" "Principle of Plant Breeding". (R.W. Allard Ed.). Jhon Willey and sons. Inc., New York.

Umakanth, A.V., Rao, S.S and Kuriakose S.V. (2006). Heterosis in landrace hybrids of post rainy sorghum (Sorghum bicolor (L.) Moench).Indian Journal of Agricultural Research,40 (2):147-150.

\section{How to cite this article:}

Meena, B.L., B.R. Ranwah, S.P. Das, H.S. Meena, S.K. Meena, R. Kumari and Anamika Nath. 2018. Assessment of Economic Heterosis in Dual Purpose Sorghum [Sorghum bicolor (L.) Moench]. Int.J.Curr.Microbiol.App.Sci. 7(07): 3196-3205. doi: https://doi.org/10.20546/ijcmas.2018.707.373 